\title{
"CONSTRUCCIÓN Y VALIDACIÓN DE UNA PRUEBA DE PROCESAMIENTO ELABORATIVO DE LA INFORMACIÓN Y DE HABILIDADES COMPOSICIONALES COMO ALTERNATIVA SUSTITUTORIA DE LA SUBPRUEBA DE LA APTITUD VERBAL EN LOS EXÁMENES DE ADMISIÓN A LA UNIVERSIDAD"
}

\author{
Manuel Miljánovich Costilla \\ Maria Atalaya Pisco \\ Alejandro Dioses Chocano \\ Rosa Huerta Rosales
}

\begin{abstract}
RESUMEN
El tema central de la investigación es la construcción y validación de contenido de una prueba de procesamiento elaborativo de la información, de corte cognitivo, con miras a ser usada en los exámenes de admisión a la universidad y en otras situaciones similares.

El procesamiento elaborativo de la información supone las habilidades de comprensión, organización y reorganización de contenidos, al servicio de la optimización de los procesos cognitivos de codificación, almacenamiento y recuperación de la información.

Tales habilidades y procesos comprenden. a su vez. las habilidades especificas de clasificación, análisis sintesis, comparación, contrastación, de discernimiento de niveles de generalidad y de secuencias causales y cronológicas, razonamiento lógico y capacidad argumentativa.

La hipótesis de trabajo subyacente en este estudio sostiene que las habilidades que mide la prueba en referencia están más directa y estrechamente relacionadas con el trabajo académico de los estudios universitarios que las habilidades medidas por la subprueba verbal de aptitud académica y que, en consecuencia, su validez predictiva del éxito en los estudios universitarios de cualquier carrera es mayor que la de esta última.

La comprobación empírica de la validez predictiva de la Prueba de Procesamiento elaborativo de la Información es materia de otro proyecto de investigación, consistente, básicamente, en un estudio longitudinal o de seguimiento de una muestra de estudiantes de diferentes carreras durante el periodo de su formación profesional.
\end{abstract}

Palabras clave: validación prueba procesamiento elaborativo de la información, habilidades composicionales

\section{ABSTRACT}

The central topic of the investigation is the construction and validation of content of a test of prosecution elaborative of the information, of court cognitive, with an eye toward being used in the admission exams to the university and in other similar situations.

The prosecution elaborative of the information supposes the abilities of understanding, organization and reorganization of contents, to the service of the optimization of the processes code cognitive, storage and recovery of the information.

Such abilities and processes understand, in/urn, the abilities specify of classification, analysis-synthesis, comparison, contrasting, of discernment of levels of generality and of causal and chronological sequences, logical reasoning and argumentative capacity

The hypothesis of underlying Work in this study sustains that the abilities that it measures the test in reference are more direct and closely related with the academic work of the university' studies that the abilities measured by the verbal subtest of academic aptitude and that, in consequence, its validity predictive of the success in the university studies of an.!' career is bigger than that of this last one.

The empiric confirmation of the validity predictive of the Test of Prosecution Elaborativo of the Information is basically matter of another project of investigation, consistent, in a longitudinal study or of pursuit of a sample of students of different careers during the period of its professional formation.

Key words: Validation, test elaborative information processing, compositional skills 
Ps. María Atalaya Pisco. Docente Asociada. Área de Psicología Organizacional UNMSM

Mg. Alejandro Dioses Chocano. Docente Asociado del Área de Psicología Educativa UNMSM

Mg. Rosa Huerta Rosales. Docente Auxiliar del área de Psicología Clínica UNMSM

La investigación realizada ha estado orientada básicamente a la construcción y validación inicial de una Prueba de Procesamiento Elaborativo de la Información, concebida desde una perspectiva cognitiva, con miras a ser usada como uno de los instrumentos de selección para el ingreso a la universidad, en mérito a que mide habilidades más estrecha y directamente ligadas a los estudios universitarios que la subprueba de Aptitud Yerbal del Scholastic Aptitude Test (SAT), vigente en San Marcos desde 1970. Por lo tanto, se hipotetiza que la prueba en referencia posee mayor capacidad predictiva que esta última, lo cual habrá de comprobarse a través de estudios longitudinales o de seguimiento ya iniciados.

PLANTEAMIENTO DEL PROBLEMA Aunque se reconocen las virtudes de la prueba de aptitud académica en lo que respecta a su relativa validez predictiva del éxito en los estudios universitarios, su validez de constructo y sus ventajas operacionales, es cierto también que desde hace ya varios años en el exterior y, en alguna medida en el país, se señalan las limitaciones de dicha prueba en los siguientes aspectos:

A. No mide habilidades composicionales, esto es, la capacidad del postulante para organizar sus ideas acerca de un determinado tema y de elaborar y redactar un informe en tomo a él, poniendo a prueba sus habilidades argumentativas y de manejo del lenguaje.

B. No mide habilidades de procesamiento elaborativo de la información en términos de codificación, almacenamiento y recuperación, según las demandas del trabajo académico y del desempeño profesional ulterior.

C. Algunos calificados tratadistas, como Robert Stemberg (1997), sostienen que el Scholastic Aptitud Test (SAT) o Prueba de Aptitud Académica, sólo predice los puntajes que los postulantes obtendrán en tests similares y pruebas educacionales, pero no las habilidades de las que realmente depende el éxito académico y profesional.

D. Numerosos profesores sanmarquinos de diferentes facultades opinan que la mayoría de los estudiantes no sabe elaborar y redactar adecuadamente sus trabajos y sus exámenes, deficiencia que atribuyen al modelo de prueba de admisión en uso.

Frente a la situación planteada, se propone la construcción de una Prueba de Procesamiento Elaborativo de la Información como alternativa de la subprueba de Aptitud Yerbal en los exámenes de admisión a la universidad y en otras situaciones similares en la que se requiere formular una predicción, con el menor error probable que sea posible, acerca del éxito o fracaso en la labor académica a desarrollar

\section{FUNDAMENTACION BIBLIOGRAFICA}

El concepto de "procesamiento de la información", invocado en el presente contexto, proviene de los enfoques dominantes en la actualidad dentro de la psicología cognitiva. Según dicho concepto, en todo aprendizaje verbal y en toda actividad intelectual o cognoscitiva están implícitos los procesos de codificación, almacenamiento y recuperación de la información. Estos procesos, a su vez, involucran las habilidades de comprensión, organización, y reorganización de los contenidos del material a aprender o a elaborar, cualesquiera sean las ciencias o disciplinas de las que se trate. Estas habilidades, a su turno, son susceptibles de desagregarse en habilidades especificas de clasificación, análisissíntesis, comparación, contrastación, de discernimiento de niveles de generalidad y de secuencias causal es y cronológicas, así como de razonamiento lógico y capacidad argumentativa. 
Una de las premisas de las que parte la presente investigación es la consideración de que las habilidades señaladas, propias del concepto amplio de "procesamiento de la información", están más directa y estrechamente ligadas a la labor académica del estudiante universitario que las habilidades medidas por las subprueba de Aptitud Verbal a través de los Ítemes de antónimos, analogías, oraciones incompletas, términos excluidos, conectores, eliminación de oraciones y plan de redacción. Excluimos expresamente de esta consideración los ítemes de comprensión de lectura por que éstos se hallan más cercanos que sus congéneres a las aludidas habilidades de procesamiento de la información

Los estudios realizados acerca del procesamiento de la información en relación con el aprendizaje escolar y académico, y su optimización, son numerosos. A título de ejemplo y en mérito a su carácter emblemático, citamos a algunos investigadores y formulamos una referencia sintética de algún aspecto de sus obras.

- Robert Schmek, a través de sus diversas obras, sostiene que la solución autodirigida de los problemas por pensadores independientes que utilizan estrategias cognoscitivas es la meta suprema de muchas formas de educación

- Dansereau y colaboradores, en la perspectiva del pensamiento de R. Gagné, generaron estrategias de aprendizaje que incluían procedimientos de reticulación con la finalidad de ayudar al estudiante a reorganizar, integrar y elaborar una tarea de lectura, así como de nodulación para interpretar, comentar y sintetizar contenidos relevantes de un determinado material de estructura compleja que se desea aprender.

- Schmek y colaboradores hallaron que las habilidades de procesamiento elaborativo de la información (que Schmek denominó procesamiento significativo) son medios decisivos para la optimización del aprendizaje académico. Dentro de dicha clase de habilidades destacó la clasificación, la comparación, la contrastación, el análisis síntesis y la capacidad para redefinir la información con los propios términos y "embellecerla" mediante la generación de analogías y ejemplos personales. Comprobó también que tales habilidades influyen en mayor medida que los "hábitos de estudio" respecto a la calidad del aprendizaje.

- David Ausubel, con su tesis central del Aprendizaje Significativo, que supone el adecuado engarce entre la estructura cognoscitiva del sujeto y el conocimiento nuevo, pone el acento en el Procesamiento Elaborativo de la Información y en un apropiado nivel de motivación.

- Joseph Novak, perfecciona y desarrolla el concepto y la técnica del mapa conceptual, el mismo que implica un alto grado el procesamiento elaborativo de la información.

\section{JUSTIFICACIÓN}

Los estudios realizados en el ámbito de la UNMSM acerca de la validez predictiva del modelo de la prueba de admisión y sus partes componentes ponen de manifiesto que las correlaciones entre la subprueba de aptitud verbal y el rendimiento académico son relativamente bajas y muy heterogéneas. Tal comprobación plantea la necesidad de sustituir la citada subprueba de aptitud verbal por otro instrumento que posea mayor capacidad predictiva del éxito en los estudios universitarios.

Las teorías cognitivas del aprendizaje y sus aplicaciones en el ámbito educativo muestran que entre los procesos de control de la memoria, son los ensayos elaborativos (opuestos a los ensayos rutinarios) los que presentan mayor analogía y mayores implicaciones con el aprendizaje académico y el trabajo intelectual universitario.

Esta investigación está orientada a responder a la demanda de contar con una prueba que posea las características señaladas, con cargo de proseguir los estudios que permitan el 
perfeccionamiento de su contenido, forma y validez predictiva.

\section{OBJETIVO}

El objetivo de la investigación es construir una prueba de procesamiento elaborativo de la información, validada en lo que respecta a contenido y forma y establecer las bases para iniciar un estudio longitudinal o de seguimiento con la finalidad de determinar su validez predictiva del éxito o fracaso en los estudios universitarios.

\section{VARIABLES}

La variable sustantiva en este estudio es el procesamiento elaborativo de la información, la misma que constituye un constructo referente a procesos del pensamiento, en el marco de las teorías cognitivas de! aprendizaje y de la memoria.

La variable controlada es el nivel de estudios de los sujetos, a saber: nivel de estudios secundarios, preparación preuniversitatia y estudios universitarios, con especificación de la carrera que se sigue.

\section{HIPÓTESIS}

Se asume como hipótesis de la presente investigación que si un instrumento de selección para el ingreso a la universidad mide las habilidades que desempeñan un papel importante en la labor intelectual típica de los estudios universitarios, como es el caso de las habilidades de procesamiento elaborativo de la información, entonces su capacidad predictiva del éxito en dichos estudios será mayor que la de un instrumento que mida habilidades sólo indirectamente relacionadas con los estudios universitarios, como es el caso de la referida subprueba de aptitud verbal.

\section{MÉTODO}

La investigación es de tipo aplicativo y está orientada a la construcción de una Prueba de Procesamiento Elaborativo de la Información, con arreglo a los métodos y normas técnicas de la construcción de instrumentos psicométricos.

La validez de contenido ha sido determinada por el juicio de los expertos que participaron en su elaboración, así como por el análisis de ítemes y la comparación de los puntajes entre niveles educativos y grupos de sujetos.

\section{MUESTRA}

La muestra es probabilística intencionada y está integrada por un total de 758 sujetos, desagregado en 167 alumnos del 5to. Año de Secundaria de zona urbano marginal, 106 de un Instituto de Secretariado, 175 de una Academia Pre - Universitaria, 116 alumnos del primer año de la Facultad de Odontología, 116 alumnos del primer año de la Facultad de Administración y 78 estudiantes del 4to. año de estudios de la Facultad de Psicología.

\section{Cuadro $\mathbf{N}^{\circ} 1$ \\ Muestra de los sujetos del estudio}

$\begin{array}{ll}\text { Alumnos del Sto. Año de Seco } & 167 \\ \text { Instituto de Secretariado } & 106 \\ \text { Academia Pre - Universitaria } & 175 \\ \text { Fac. de Odontología de la UNMSM } & 116 \\ \text { Fac. de Administración de la UNMSM } & 116 \\ \text { Fac. de Psicología. de la UNMSM } & 78 \\ \text { TOTAL } & \mathbf{7 5 8}\end{array}$




\section{INSTRUMENTO}

La forma final de la prueba consiste en dos temas que se anuncian como subtítulos y van seguidos de un conjunto de párrafos numerados, que se refieren a diversos aspectos del tema, en diferentes niveles de generalidad.

El primer tema es más corto y de menor dificultad que el segundo con la finalidad de no desalentar a los sujetos y encarrilarlos en la resolución de la prueba. La tarea consiste en responder a cinco preguntas de selección múltiple por cada tema, haciendo un total de diez preguntas.

En los primeros ítemes de ambos temas, se trata de identificar, en el conjunto de los párrafos, aquellos que por su contenido están relacionados con el subtema propuesto en el enunciado del ítem y, luego, responder eligiendo la alternativa que contenga los números que designan dichos párrafos.

Estos ítemes demandan habilidades de comprensión, análisis y discriminación fina de contenidos.

En los ítemes posteriores no sólo se trata de identificar párrafos relacionados con un subtema sino, además, de organizar los párrafos seleccionados según determinados criterios y establecer entre ellos relaciones de tipo argumentativo a favor o en contra de una tesis, de un hecho o una posibilidad.

En estos ítemes intervienen habilidades de comprensión, de razonamiento lógico, clasificación, análisis-síntesis, discernimiento de niveles de generalidad, de secuencias causal es y cronológicas, así como de comparación, contrastación, valoración y reorganización argumenta ti va.

En primer tema es Estrés y Trabajo y comprende 16 párrafos. El segundo es el Petróleo y la contaminación atmosférica y comprende 31 párrafos.

Los principales pasos secuenciales en la construcción de la prueba fueron los siguientes:

- Formulación de la tabla de especificaciones, luego de examinar diversas alternativas respecto a la estructura de la prueba.

- Elaboración de proyectos de ítemes, según la tabla de especificaciones

- Discusión grupal de ítemes, a cargo del equipo de investigación. Se retuvieron y afinaron los ítemes aprobados por el grupo y se elaboraron otros proyectos de ítemes destinados a reemplazar a los desechados.

- Ensamblaje de la prueba con los ítemes aprobados por el grupo y con arreglo a la tabla de especificaciones.

- Revisiones sucesivas de la prueba hasta lograr su versión apriorísticamente final

- Pre-test y reajustes consecuentes, tanto en el contenido de algunos ítemes, como en su forma y en las instrucciones.

- Versión definitiva.

La confiabilidad de la prueba, en términos de consistencia interna, fue detern1inada mediante la fórmula de Spearman- Brown y alcanzó el valor de 0.78, el mismo que es evidentemente satisfactorio.

\section{PROCEDIMIENTOS}

La prueba se aplicó a los sujetos integrantes de la muestra, asegurando previamente la existencia de condiciones favorables para este efecto. Se puso énfasis en la motivación de los sujetos y en la cabal comprensión de las instrucciones. Se cuidó de absolver todas las dudas antes de empezar la resolución de la prueba y de detectar, mediante discretas observaciones, la persistencia de algún malentendido. 
Bajo tales condiciones, las aplicaciones se realizaron adecuadamente. La calificación de los protocolos de respuesta se efectuó por contrastación con la clave y se determinaron los puntajes parciales y totales.

Se desecharon los protocolos de los sujetos que no habían concluido la prueba. El tiempo promedio de aplicación fue de 40 minutos.

\section{PROCESAMIENTO ESTADÍSTICO}

El procesamiento estadístico de los puntajes comprendió tres rubros, a saber:

1. Estimación del grado de aproximación de la distribución de nuestros puntajes a la curva normal de probabilidades, mediante el cálculo de la asimetría y de la curtosis.

2. Cálculo de las medias y las desviaciones estándar de cada uno de los grupos y de la muestra total. (Cuadro $\mathrm{N}^{\circ} 2$ )

3. Comparación de los diferentes grupos definidos por los grados y tipos de estudios.

En primera instancia se empleó un modelo de análisis de varianza de un criterio de clasificación. El hecho de que el valor del estadístico F resultara significativo al nivel del 0.05 , determinó que, en segunda instancia, efectuaremos todas las compa-raciones posibles entre pares de grupos, mediante el estadístico " $t$ ' de Student.

4. El análisis de ítemes comprendió las siguientes determinaciones respecto a cada ítem:

a) Determinación del índice de dificultad.

b)Determinación del índice de discriminación.

c) Examen y evaluación del funcionamiento de las alternativas

5. Determinación del coeficiente de confiabilidad.

\section{RESULTADOS}

\section{Análisis cuantitativo y cualitativo}

Hay una marcada gradiente en el nivel de dificultad de los ítemes (Cuadro $\mathrm{N}^{\circ} 4$ ), correlativa al nivel de complejidad del procesamiento elaborativo de la información.

En los ítemes que demandan sólo las habilidades de comprensión e identificación los puntajes son notoriamente más altos que los ítemes que requieren el despliegue de habilidades más complejas (que suponen a las simples, como base), tales como razonamiento lógico, discernimiento de niveles de generalidad y de secuencias causales o cronológicas, valoración y elaboración argumentativa. 
Cuadro 2

Medias aritméticas $(X)$ y Desviaciones estándar $(D S)$ de los puntajes por grupos

\begin{tabular}{|l|c|c|}
\hline \multicolumn{1}{|c|}{ Grupos } & X & DS \\
\hline Alumnos del 5to de Secundaria & 3.823 & 1.999 \\
Inst. de Secretariado & 7.12 & 1.836 \\
Acad. Pre Universitaria & 7.576 & 1.731 \\
Fac. De Odontología UNMSM & 7.933 & 1.645 \\
Fac de Administración UNMSM & 7.35 & 1.706 \\
Fac. de Psicología UNMSM & 8.677 & 1.113 \\
\hline
\end{tabular}

Cuadro 3

Comparación de las medias de los puntajes en la prueba de procesamientos de la información mediante el estadistico " $t$ " de student

\begin{tabular}{|c|c|c|c|c|c|c|}
\hline & \begin{tabular}{|c|} 
5to \\
secundaria \\
$\mathrm{N}: 124$ \\
$\mathrm{X}: 3.823$ \\
$\mathrm{Ds}: 1999$ \\
\end{tabular} & $\begin{array}{c}\text { Instituto } \\
\text { Secret. } \\
\text { N: } 106 \\
\text { X:7.12 } \\
\text { Ds: } 1.836\end{array}$ & $\begin{array}{c}\text { Academia } \\
\text { Pre-Univ. } \\
\text { N: } 175 \\
\text { X: } 7.576 \\
\text { Ds: } 1.731\end{array}$ & $\begin{array}{c}\text { Odontol. } \\
\text { N:116 } \\
\text { X:7.933 } \\
\text { Ds: } 1.645\end{array}$ & $\begin{array}{c}\text { Administ. } \\
\text { N:116 } \\
\mathrm{X}: 7.35 \\
\text { Ds: } 1.706\end{array}$ & $\begin{array}{c}\text { Psicol. } \\
\text { N:121 } \\
\text { X:8.677 } \\
\text { Ds:1.213 }\end{array}$ \\
\hline $\begin{array}{c}\text { 5to.Sec. } \\
\mathrm{N}: 124 \\
\mathrm{X}: 3.823 \\
\mathrm{Ds}: 1999\end{array}$ & & $\begin{array}{l}\text { " } t " c: 7.02 \\
\text { sig.:0.01 }\end{array}$ & $\begin{array}{l}\text { “t”c:9.59 } \\
\text { sig.:0.01 }\end{array}$ & $\begin{array}{l}\text { " } t \text { "c :8.43 } \\
\text { sig.:0.01 }\end{array}$ & $\begin{array}{l}\text { " } t \text { "c:7.77 } \\
\text { Sig.:0.01 }\end{array}$ & $\begin{array}{l}\text { " } t \text { "c:9.58 } \\
\text { Sig.:0.01 }\end{array}$ \\
\hline $\begin{array}{c}\text { Ins.Secret. } \\
\text { N:124 } \\
X: 3.823 \\
\text { Ds:1999 } \\
\end{array}$ & & & $\begin{array}{l}\text { “ } t \text { "c.:1.52 } \\
\text { No Sig. }\end{array}$ & $\begin{array}{l}\text { " } t \text { "c:2.36 } \\
\text { Sig.:0.05 }\end{array}$ & $\begin{array}{l}\text { “t”c:0.68 } \\
\text { NoSig. }\end{array}$ & $\begin{array}{l}\text { " } t \text { "c:3.90 } \\
\text { Sig.:0.01 }\end{array}$ \\
\hline $\begin{array}{c}\text { Pre-Univ. } \\
\mathrm{N}: 124 \\
\mathrm{X}: 3.823 \\
\text { Ds:1999 } \\
\end{array}$ & & & & $\begin{array}{l}\text { “t”c:1.31 } \\
\text { No Sig. }\end{array}$ & $\begin{array}{l}\text { “t”c:0.83 } \\
\text { No Sig. }\end{array}$ & $\begin{array}{l}\text { " } t \text { "c:4.04 } \\
\text { Sig.:0.01 }\end{array}$ \\
\hline $\begin{array}{c}\text { Odontol. } \\
\text { N:124 } \\
\mathrm{X}: 3.823 \\
\text { Ds:1999 }\end{array}$ & & & & & $\begin{array}{l}\text { " } t \text { "c:1.89 } \\
\text { No Sig. }\end{array}$ & $\begin{array}{l}\text { " } t \text { "c:2.88 } \\
\text { Sig.:0.01 }\end{array}$ \\
\hline $\begin{array}{c}\text { Administ. } \\
\mathrm{N}: 124 \\
\mathrm{X}: 3.823 \\
\text { Ds:1999 } \\
\end{array}$ & & & & & & $\begin{array}{l}\text { " } t \text { "c:5.40 } \\
\text { Sig.:0.01 }\end{array}$ \\
\hline $\begin{array}{c}\text { Psicol. } \\
\text { N:124 } \\
\text { X:3.823 } \\
\text { Ds:1999 }\end{array}$ & & & & & & \\
\hline
\end{tabular}

" $t$ " $c=$ valor de la " $t$ calculada" valores de la " $t$ " tabulada

Signific:0.01=Significativa al nivel del 0.01 al $0.01: 2.02$

Signific:0.05= Significativa al nivel del 0.05 al 0.05: 2.71

No Signific $=$ No significativa al nivel del 0.05 


\section{Cuadro $\mathrm{N}^{\circ} 4$ \\ Proyecto 200-prueba de procesamiento de la información-funcionamiento de las alternativas}

\begin{tabular}{|c|c|c|c|c|c|}
\hline & $\mathbf{A}$ & $\mathbf{B}$ & $\mathbf{C}$ & $\mathbf{D}$ & $\mathbf{O}$ \\
\hline 1 & $16.37 \%$ & $1.10 \%$ & $65.04 \%$ & $8.85 \%$ & $8.63 \%$ \\
2 & $8.41 \%$ & $70.35 \%$ & $11.10 \%$ & $2.88 \%$ & $6.41 \%$ \\
3 & $6.20 \%$ & $1.11 \%$ & $4.20 \%$ & $86.28 \%$ & $2.21 \%$ \\
4 & $81.86 \%$ & $2.65 \%$ & $6.42 \%$ & $2.88 \%$ & $6.19 \%$ \\
5 & $11.50 \%$ & $3.10 \%$ & $77.65 \%$ & $2.65 \%$ & $5.09 \%$ \\
6 & $5.31 \%$ & $57.08 \%$ & $7.30 \%$ & $20.35 \%$ & $9.95 \%$ \\
7 & $13.93 \%$ & $3.76 \%$ & $4.87 \%$ & $71.90 \%$ & $5.53 \%$ \\
8 & $10.18 \%$ & $4.87 \%$ & $71.0 \%$ & $6.86 \%$ & $7.08 \%$ \\
9 & $4.20 \%$ & $13.94 \%$ & $6.41 \%$ & $67.70 \%$ & $7.74 \%$ \\
10 & $61.95 \%$ & $11.06 \%$ & $8.18 \%$ & $8.85 \%$ & $9.95 \%$ \\
\hline
\end{tabular}

\section{COMPARACIONES ENTRE GRUPOS}

Los resultados de la comparación de los medios de los grupos entre sí, mediante el estadístico "t" de Student, (Cuadro $N^{\circ} 3$ ) muestran que los estudiantes del 5to año de educación secundaria son superados netamente por todos los demás grupos, al nivel de significación del 0.01 .

Las estudiantes de Secretariado superan categóricamente a los estudiantes de secundaria; no presentan diferencias significativas con los estudiantes pre-universitarios ni con los de la Facultad de Administración y son superadas por los estudiantes de las facultades de Odontología y de Psicología, al 0.05 y 0.01 de significación, respectivamente.

Los estudiantes de la academia pre-universitaria superan, como se dijo, a los estudiantes de secundaria; no presentan diferencias significativas con las estudiantes de secretariado ni con los de las facultades de odontología y de administración y sólo son superados por los estudiantes de psicología, al 0.01 de significación.

Los estudiantes de la facultad de Odontología superan, como se señaló, a los estudiantes de secundaria y a las estudiantes de Secretariado, no presentan diferencias significativas con los estudiantes preuniversitarios ni con los de la Facultad de Administración y sólo son superados por los estudiantes de la Facultad de Psicología, al nivel del 0.01.

Las estudiantes de la Facultad de Administración superan, como se expresó, a los estudiantes de secundaria, no presentan diferencias significativas con los estudiantes de Secretariado, ni con los estudiantes preuniversitarios ni con los estudiantes de Odontología y sólo son separados por los estudiantes de Psicología, al nivel 0.01 . 
Finalmente, los estudiantes de la Facultad de Psicología superan categóricamente, al nivel de significación del 0.01, a todos los otros grupos.

Destacan nítidamente dos grupos contrapuestos: los estudiantes del 5to año de secundaria de una zona urbano-marginal y los estudiantes del sexto semestre de la Facultad de Psicología que presentan, respectivamente, el más bajo y el más alto rendimiento en la Prueba de Procesamiento Elaborativo de la Información.

\section{El grado de dificultad y el poder discriminativo de los itemes en los grupos extremos de rendimiento y en el caso especial del grupo pre-universitario}

\section{GRUPO: Estudiantes del5to. de Secundaria de zona urbana-marginal}

Para esta submuestra ocho de los diez ítems administrados presentaron un elevado índice de dificultad. Sólo los Ítemes tres $(0.48)$ y cuatro(0.57) presentaron un índice de dificultad promedio. Por otro lado, se encontró que los ítemes tres (0.72), cuatro (0.66), cinco (0.77) Y ocho (0.55), evidenciaban adecuados índices de discriminación, por lo que colegimos que los Ítemes cinco y ocho presentan condiciones deseables, tanto en el ámbito de dificultad, como en el nivel de discriminación. Cabe remarcar que este grupo de alumnos carece de apropiadas oportunidades para desarrollar sus habilidades cognitivas, debido a su precaria situación económica, además de no tener adecuados modelos ni estímulos para desarrollar sus niveles de pensamiento.

\section{GRUPO: Estudiantes de Academia PreUniversitaria (Grupo de alto rendimiento académico).}

Como en el caso de los alumnos universitarios, para el grupo integrado por los alumnos preuniversitarios, los ítemes tuvieron un bajo nivel de dificultad, apreciándose que sólo los ítemes seis (0.37) y diez ( 0.35 ) tienen moderados índices de dificultad.

En este caso los resultados se explican porque se trata de un grupo de élite en dicho centro de estudios, al que sólo se accede luego de un riguroso proceso de evaluación que incluye, además de razonamiento matemático y verbal, un dominio a nivel de excelencia de las asignaturas de ciencias. Los que son seleccionados reciben un entrenamiento de $1 \mathrm{O}$ a 12 horas diarias con el objetivo de ingresar a las universidades ocupando los primeros puestos. Los antecedentes educativos de todos estos alumnos los ubican, en sus respectivos colegios, ocupando los tres primeros lugares de su promoción a lo largo de sus estudios secundarios.

\section{GRUPO: Estudiantes universitarios de psicología.}

El análisis de los resultados muestra que, para este grupo, la totalidad de ítemes resultó con un índice de dificultad sumamente bajo. Esto puede ser explicado porque la prueba se diseñó pensando en alumnos que han concluido la educación secundaria, mientras que la muestra examinada se encuentra cursando el sexto semestre de la especialidad de psicología, además de ser una carrera en la que los alumnos, a este nivel de avance, ya han recibido un entrenamiento fuerte en razonamiento verbal, comprensión lectora y técnicas de estudio, lo que individualmente los pone en condiciones muy favorables para resolver ítemes de esta índole.

Es importante resaltar que los índices de discriminación también fueron bastantes bajos llegando al extremo de ser cero para los ítems tres y cuatro, a pesar de ello, cabe resaltar que en los ítems uno, siete y diez sí se alcanzaron niveles de discriminación altos, que sin embargo, son de poca utilidad dados los resultados generales alcanzados por la muestra. 


\section{INTERPRETACIÓN DE LOS RESULTADOS}

La interpretación de los resultados del procesamiento estadístico de los datos, a la luz del marco teórico-conceptual acotado, nos permite derivar deductivamente las siguientes apreciaciones:

1. La confiabilidad de la prueba, en términos de consistencia interna, determinada mediante la fórmula de Spearman-Brown, alcanza el valor de 0.78 , el mismo que es evidentemente satisfactorio.

2. La comparación de los puntajes entre los grupos definidos por los tipos y grados de estudios, mediante el estadístico " $F$ " del análisis de varianza, y, luego, entre pares de los diferentes grupos mediante el estadístico " $t$ " de Student, revela que el desarrollo de las habilidades de procesamiento de la información presenta una gradiente muy marcada desde el 5to año de educación hasta el nivel de los estudios universitarios avanzados.

El rasgo más destacado de dicha gradiente es el bajo nivel, acentuada-mente inferior al resto de la muestra, que presentan los estudiantes del 5to año de educación secundaria, lo cual es indicativo de la crisis de este nivel educativo en cuanto al desarrollo de habilidades de clasificación, síntesis-análisis, comparación, contrastación, razonamiento lógico, de argumentación y de reorganización de la información.

Lo anteriormente señalado adquiere mayor relieve si se tiene en cuenta que los puntajes obtenidos por los estudiantes de las academias preuniversitarias superan categóricamente, al nivel de significación del 0.0 1, a los obtenidos por los estudiantes del 5to año de secundaria, hecho que confirma la conjetura de que existe un marcado y critico déficit formativo en el área cognoscitiva en la educación secundaria. Pone también de manifiesto el hecho de que la crisis de la educación secundaria, cuyos factores causales son múltiples, ha contribuido a institucionalizar el nivel artificioso y mercantilista de la "academia pre-universitaria", como condición para que los egresados de la educación secundaria puedan acceder a la universidad.

3. El hecho de que los estudiantes de Psicología superen netamente a los de Odontología y Administración tienen importantes implicancias, a saber:

a. En los procesos de admisión de los últimos 8 años, cuando menos, los ingresantes a Odontología superan a los de Administración y a los de Psicología en el puntaje de la prueba de admisión. El orden de los promedios de dichos puntajes es Odontología, Administración y Psicología.

b. Los estudiantes de Psicología que forman parte de la muestra del presente estudio cursan el sexto semestre de estudios, en tanto que los de Administración y de Odontología cursan el 1er año

C. Las anteriores consideraciones nos permiten sostener:

1) Que la prueba de admisión a San Marcos y la Prueba de Procesamiento Elaborativo de la Información aplicada en el presente estudio miden habilidades y contenidos diferentes, aunque no se puede descartar que exista entre ambas una zona menor de intersección.

2) Que las habilidades de procesamiento de la información son susceptibles de desarrollo a través del aprendizaje, como lo demuestra la neta superioridad de los puntajes de los estudiantes del sexto semestre de estudios universitarios (Psicología) sobre aquellos que cursan el ler. año ( Odontología y Administración).

Respalda esta hipótesis la constatación de la categórica superioridad de los puntajes de los estudiantes de academias preuniversitarias sobre los estudiantes del 5to. año de 
secundaria.

\section{CONCLUSIONES}

1. El análisis de los resultados del procesamiento de los datos muestra que la prueba construida posee adecuada validez de contenido, en el sentido de medir realmente las habilidades de procesamiento de la información consignadas en el marco teórico.

Dos hechos respaldan este aserto, a saber:

a) El juicio de expertos, representado en este caso por los miembros del equipo constructor, los mismos que ostentan la condición de psicólogos altamente especializados y expertos en construcción de pruebas, quienes en cada etapa analizaron los reactivos tentativos, los reajustaron o desecharon y sustituyeron, etc, hasta el logro de la versión final, teniendo presente en todo momento el marco teórico acotado y los objetivos propuestos.

b) La estructura de la prueba, a la luz de los resultados de sus aplicaciones y del análisis de los puntajes, esto es, de la constatación empírica de sus características, presenta propiedades adecuadas en términos de Índice de Dificultad, Índice de Discriminación, del funcionamiento de las alternativas y de la capacidad de diferenciar rendimientos en función del nivel de los estudios académicos.

2. De las realizaciones de la presente investigación se desprenden tres necesidades

a) Determinar la validez de constructo de la prueba mediante la correlación de sus puntajes con algún criterio externo comprobadamente válido que, dicho a riesgo de redundar, mida las mencionadas habilidades de procesamiento de la información.

b) Determinar la validez predictiva de la prueba respecto al éxito o fracaso de los estudios universitarios, a través de un estudio longitudinal o de seguimiento de muestras de ingresantes a diversas facultades que permita la correlación, por un lado, de los puntajes en la prueba de procesamiento de la información aplicada antes del inicio de clases con los promedios ponderados de las notas al término de cada uno de los cuatro primeros semestres académicos y comparar dichos coeficientes con los otros, resultantes de similar operación, pero en este caso entre los puntajes en la subprueba de aptitud verbal del examen de admisión y los promedios ponderados en las mencionadas notas de cursos correspondientes al periodo indicado. Eso permitirá comparar, en base a evidencias empíricas controladas, la validez predictiva de las dos pruebas en referencia.

\section{Cuadro $\mathbf{N}^{\circ} 5$}

\section{Baremos o normas referenciales de la prueba de procesamiento elaborativo de}

\section{la información}

Con submuestras de estudiantes universitarios y estudiantes del quinto año de educación secundaria se han elaborado baremos provisionales o referenciales, con cargo de desarrollarlos y perfeccionarlos posteriormente, en base a muestras más amplias y representativas de las poblaciones a las que se destinan. 


\section{ESTUDIANTES UNIVERSITARIOS}

\begin{tabular}{|l|r|c|c|}
\hline $\begin{array}{c}\text { Puntaje } \\
\text { bruto }\end{array}$ & \multicolumn{3}{|c|}{$\begin{array}{c}\text { Puntajes } \\
\text { transformados }\end{array}$} \\
\hline \multirow{4}{*}{2} & Ptje. z & Ptje. T & Stanford-Binet \\
\cline { 2 - 4 } 3 & -2.50 & 25.0 & 60.00 \\
4 & -2.19 & 28.1 & 64.96 \\
5 & -1.81 & 31.9 & 71.04 \\
6 & -1.26 & 37.4 & 79.84 \\
7 & -0.64 & 43.6 & 89.76 \\
8 & -0.22 & 47.8 & 96.48 \\
9 & 0.25 & 52.5 & 104.00 \\
10 & 0.87 & 58.7 & 113.92 \\
& 1.81 & 68.1 & 128.96 \\
\hline
\end{tabular}

\section{ESTUDIANTES DEL 5to. AÑO DE EDUCACION SECUNDARIA}

\begin{tabular}{|c|c|c|c|}
\hline $\begin{array}{c}\text { Puntaje } \\
\text { bruto }\end{array}$ & \multicolumn{3}{|c|}{$\begin{array}{c}\text { Puntajes } \\
\text { transformados }\end{array}$} \\
\hline & Ptje. z & Ptje. T & Stanford-Binet \\
\cline { 2 - 4 } 1 & -2.83 & 21.7 & 54.72 \\
2 & -1.84 & 31.6 & 70.56 \\
3 & -0.82 & 41.8 & 86.88 \\
4 & -0.32 & 46.8 & 94.88 \\
5 & 0.15 & 51.5 & 102.40 \\
6 & 0.59 & 55.9 & 109.44 \\
7 & 1.02 & 60.2 & 110.20 \\
8 & 1.40 & 64.0 & 122.40 \\
9 & 1.89 & 68.9 & 130.24 \\
\hline
\end{tabular}

\section{BIBLIOGRAFIA}

Sternberg. Robert (1997). La inteligencia exitosa, B.Aires: Paidos.

Sternberg, Robert (1996). Discurso ante la Asoc. Norteamericana de Investigación Educacional, Santiago, Universidad de Chile.

Novak, Joseph (1998). Conocimiento y aprendizaje, Madrid: Alianza Editorial. Ausubel, D; Novak, J y Hanessian, H. (1998). Psicología educativa, México: Trillas. Puente Ferreras, A. et al (1998). Cognición y aprendizaje, Madrid: Pirámide. Muñiz, José (Coordinador) (1996). Psicometría Madrid: Universitas.,

Miljánovich Castilla, Manuel; Lopez Cruz, Roxana; Quesada Castillo, Félix y Bernui B., Juan (1998). Análisis de los resultados de los procesos de admisión 1996,1997 y 1998 en relación con variables psicológicas, pedagógicas y sociales. Proyecto de investigación 81801081, UNMSM, LIMA.

Miljánovich c., Manuel; MÁLAGA M, Orestes y otros. (1997) Estudio de la validez predictiva de la prueba de admisión a la UNMSM, LIMA. OCA.

Miljánovich C., Manuel. (1999). Relación entre la inteligencia, el rendimiento académico y la comprensión de lectura en el campo educativo, Tesis para optar el grado académico de Doctor en Educación, FACULTAD DE EDUCACIÓN UNMSM.

Carroll, J. B. (1980). Relaciones de diferentes individuos en tareas psicométricas y cognoscitivas experimentales, Universidad de Carolina del Norte. 